\title{
Early Tracheostomy Suture Removal for Better Swallowing? A Case Report and Literature Review
}

\author{
E Berryhill McCarty ${ }^{1 *}$, Liuba Soldatova ${ }^{2,3}$, Kyle Lees ${ }^{3}$, Natasha Mirza $^{2,3}$, and Randy Dubin ${ }^{2,3}$ \\ ${ }^{1}$ Perelman School of Medicine at the University of Pennsylvania, Philadelphia, Pennsylvania, Philadelphia, USA \\ ${ }^{2}$ Department of Otorhinolaryngology-Head and Neck Surgery, University of Pennsylvania Health System, \\ Philadelphia, Pennsylvania, Philadelphia, USA \\ ${ }^{3}$ Division of Speech Language Pathology, Good Shepherd-Penn Partners, Hospital of the University of \\ Pennsylvania, Philadelphia, USA

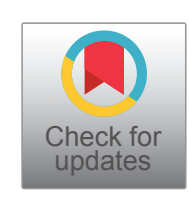

\begin{abstract}
Objectives: To draw attention to the impact of prolonged tracheostomy suture retention on post-tracheostomy swallow dysfunction, we present a clinical case of improved dysphagia following removal of tracheostomy sutures in a woman who required tracheostomy for acute onset respiratory failure.

Methods: Case report and literature review.

Results: Here we describe the case of a 60 -year-old female who presented with progressively worsening shortness of breath requiring an urgent open tracheostomy. Pre-tracheostomy Fiberoptic Endoscopic Evaluation of Swallow (FEES) revealed normal swallow function. FEES conducted three days after tracheostomy, with sutures still in place, revealed moderate oropharyngeal dysphagia with silent aspiration. Repeat FEES conducted eight days after tracheostomy, and after suture removal, showed functional oropharyngeal swallow without penetration or aspiration. Literature review revealed no prior studies that formally examine the effect of early tracheostomy suture removal on post-tracheostomy swallowing function.
\end{abstract}

Conclusions: Although other variables such as time and further clinical improvement may have contributed to the resolution of post-tracheostomy dysphagia in this patient, the case certainly raises a question of whether or not early tracheostomy suture removal can improve post-tracheostomy swallowing. In addition to surgical dissection and the tracheostomy tube itself, the sutures may anchor hyolaryngeal excursion by attaching to superficial infrahyoid muscles and underlying soft tissues, resulting in further reduced laryngeal elevation and anterior movement. This can impact both swallowing safety and efficiency. A formal study evaluating the effect of tracheostomy suture removal on post-tracheostomy dysphagia resolution is necessary. Such a study would inform the creation of uniform guidelines for appropriate timing of tracheostomy suture removal.

\section{Keywords}

Tracheostomy, Tracheostomy Sutures, Dysphagia, Swallowing, Fiberoptic Endoscopic Evaluation of Swallow

\section{Introduction}

Post-tracheostomy dysphagia is common due to patient related factors such as underlying illness, pre-existing co-morbidities, intensive care unit acquired weakness, mental status changes, and prolonged or multiple intubations [1]. Potential mechanical and physiological effects of tracheostomy on swallowing have also been identified. These include reduced laryngeal elevation and anterior movement (especially if tracheostomy cuff remains inflated), reduced laryngeal sensation, inefficient cough, and lack of subglottic air pressure/ reduced airway closure. The potential hyolaryngeal anchoring effects of a tracheostomy can lead to poor airway protection and reduced upper esophageal sphincter opening [1-3].
Different institutions and surgical specialties have variable protocols in place for tracheostomy tube management,

*Corresponding author: E Berryhill McCarty, MSHCPM, MS Perelman School of Medicine at the University of Pennsylvania, Hospital of the University of Pennsylvania, 3400 Civic Center Blvd, Philadelphia, PA 19104, USA, Tel: 412-613-6389, E-mail: elizabeth.mccarty@pennmedicine.upenn.edu

Accepted: November 23, 2020

Published online: November 25, 2020

Citation: McCarty EB, Soldatova L, Lees K, et al. (2020) Early Tracheostomy Suture Removal for Better Swallowing? A Case Report and Literature Review. Otolaryngol Res Rev 3(1):60-63 
including timing of cuff deflation, the downsizing and changing to a cuffless tracheostomy tube, and timing of suture removal. These tracheostomy tube protocols affect the timing of swallowing evaluation, therapy, and return to an oral diet. Numerous clinical bedside swallow evaluations (CBSEs) at our institution reveal reduced hyolaryngeal elevation to palpation in tracheostomy patients with sutures in place, resulting in potential dysphagia and leading to delays in re-initiation of an oral diet. It is also not unusual to identify reduced superior and anterior hyoid movement during modified barium swallow studies in patients with sutured tracheostomy tubes. To draw attention to this potential contributing (yet easily correctible) factor in post-tracheostomy dysphagia, we present a clinical case to highlight the issue of tracheostomy suture retention and the possible impact these sutures have on post-tracheostomy swallowing function.

\section{Case Report}

A 60-year-old female with a history of obesity, type 2 diabetes, peripheral vascular disease, recent COVID19 diagnosis, and asthma presented with progressively worsening shortness of breath. Five months previously she had been hospitalized for status asthmaticus, requiring multiple intubations (intubated for fourteen days initially and then for three days after re-intubation). Fiberoptic Endoscopic Evaluation of Swallow (FEES) completed post-extubation revealed functional oropharyngeal swallow with no aspiration and mild right vocal fold weakness, but overall intact vocal fold mobility. She reported no symptoms of dysphagia or dysphonia and was able to tolerate a regular diet. Three months after her initial intubation, she was incidentally found to have stenosis of the trachea and left main stem bronchus on computed tomography (CT) scan for which she underwent two tracheal dilations at an outside hospital.

A few weeks after these tracheal dilations, she was readmitted to our institution with poor oxygenation requiring intubation. The initial attempt to intubate by anesthesia with a 4-0 endotracheal (ET) tube was unsuccessful. While the patient was bag-mask ventilated, the decision was made to proceed with an urgent open tracheostomy. The procedure was performed in the operating room by an experienced otolaryngologist. After a vertical anterior neck incision, the trachea was exposed and an incision was made between the second

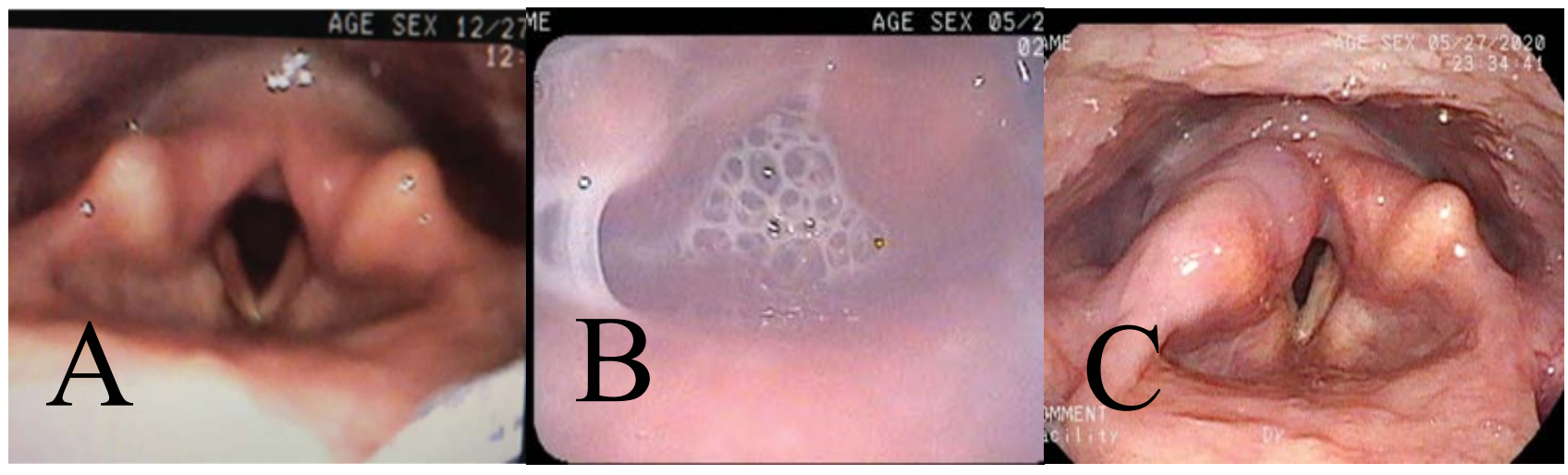

Figure 1: Fiberoptic endoscopic evalution of swallowing trend A) 12/27/19 - No tracheostomy/No Sutures: No aspiration; B) 5/22/20 - Tracheostomy/Sutures Present: Aspiration thin liquid (milk); C) 5/27/20 - Tracheostomy/No Sutures: No aspiration.

Table 1: Clinical timeline.

\begin{tabular}{|l|l|}
\hline Date & Event \\
\hline $11 / 8 / 19-11 / 22 / 19$ & Intubated for Status Asthmaticus at the outside hospital \\
\hline $11 / 30 / 19-12 / 2 / 19$ & Re-intubated for increasing O2 requirements \\
\hline $12 / 27 / 19$ & $\begin{array}{l}\text { FEES with functional oropharyngeal swallow } \\
\text { No Tracheostomy, No Tracheostomy Sutures }\end{array}$ \\
\hline $3 / 10 / 20$ & Tracheal stenosis diagnosed via incidental finding on CT scan \\
\hline $3 / 17 / 20$ and $3 / 27 / 20$ & Tracheal Dilations performed \\
\hline $5 / 19 / 20$ & Urgent Open Tracheostomy \\
\hline $5 / 22 / 20$ & FEES with moderate oropharyngeal dysfunction \\
\hline $5 / 23 / 20$ & Tracheostomy, Tracheostomy Sutures \\
\hline $5 / 27 / 20$ & Trach sutures removed \\
\hline & FEES with functional oropharyngeal swallow \\
\hline
\end{tabular}

${ }^{*}$ FEES: Fiberoptic Endoscopic Evaluation of Swallow. 
and third tracheal rings. The area of tracheal stenosis was dilated with an endotracheal tube. A 6-0 cuffed Shiley tracheostomy tube was placed and secured to the skin with prolene sutures and tracheostomy ties.

Two days after tracheostomy procedure, a speech and swallowing evaluation was requested per our institutional protocol. The patient was observed to have reduced hyolaryngeal elevation. Three days post-tracheostomy, while tracheostomy sutures were still in place, a FEES demonstrated moderate oropharyngeal dysphagia with silent aspiration of thin liquids, high penetration with solids, and intermittent penetration with pureed and nectar thick liquids. There was also evidence of silent aspiration of secretions noted with reduced bilateral vocal fold motion. She was deemed unsafe for oral diet at that time. On post-op day eight, four days after tracheostomy suture removal, repeat FEES demonstrated functional oropharyngeal swallow with only one episode of deep penetration of thin liquids, but no penetration or aspiration on subsequent trials, and overall improved secretion management (Figure 1). A regular diet was initiated and was tolerated without issue. The complete timeline is presented in Table 1.

\section{Discussion}

At many institutions, Speech and Language Pathology (SLP) protocols dictate evaluation of swallowing function once the patient is transitioned to a humidified tracheostomy collar, and CBSEs or instrumental swallow evaluations (such as FEES or modified barium swallow studies) are performed prior to tracheostomy suture removal. Anecdotally, CBSEs at our institution reveal dramatically reduced hyolaryngeal excursion to palpation when the evaluation is done while the tracheostomy sutures are in place, with improvement following suture removal.

In normal swallowing, superior and anterior displacement of the hyolaryngeal complex are necessary for airway protection and bolus transport to the esophagus via epiglottic inversion and opening of the upper esophageal sphincter (UES) [3]. Superior displacement of the hyoid is more important to the closure of the laryngeal vestibule, and anterior displacement is associated with the pulling motion on the cricopharyngeus muscle and opening of the UES [3]. Inadequate hyolaryngeal excursion (HE) may result in incomplete airway closure as well as reduced UES opening, leading to pharyngeal stasis, overflow of pharyngeal contents into the unprotected glottic commissure, and penetration or aspiration [4]. The infrahyoid muscles (IHM) play an important role in hyolaryngeal excursion [5]. The IHMs are retracted or dissected off of the trachea during tracheostomy procedure, which could potentially lead to post-tracheostomy swallowing dysfunction [6]. The scarcity of literature describing the effect of tracheostomy sutures on post-tracheostomy dysphagia makes it difficult to evaluate the effect of tracheostomy sutures on post-tracheostomy swallowing since some authors argue that the tethering of the infrahyoid muscles by the tracheostomy tube itself reduces hyolaryngeal elevation [7]. Recent systematic reviews refuted this notion and found no significant differences in hyolaryngeal excursion in patients with and without tracheosto- my $[7,8]$. These studies did not include patients with sutured tracheostomy tubes, and after conducting a literature search, we did not find any other studies that looked at the effect of tracheostomy sutures on post-tracheostomy swallowing.

Suture placement is viewed as a preventative measure to avoid accidental decannulation [9]. A study by Dixon, et al. found that suture removal, which had traditionally been conducted 7-10 days post-operatively, could be safely and effectively done on post-operative day 5 , limiting tracheostomy-related pressure ulcers [10]. A similar study by O'Toole, et al. recommended that removal of tracheostomy sutures after 7 days was optimal in reducing pressure injury [11]. Similar to the variability in timing of suture removal, different institutions also have wide variability in protocols for when swallowing evaluations should be initiated.

The clinical case described here suggests an observable difference in post-tracheostomy swallowing as documented by the pre- and post-suture removal FEES. Of note, as a result of the vertical incision required in this patient due to her known history of tracheal stenosis, it is possible that the surrounding strap muscles experienced more extensive retraction and dissection during the operation, which could have impacted the degree of post-operative scarring and tethering of the hyolaryngeal complex. Nevertheless, this clinical case raises a question of whether tracheostomy sutures can have a significant effect on post-tracheostomy swallowing leading to poor swallow function and delayed diet advancement. It is not uncommon for patients to quickly wean off ventilators following tracheostomy. Given the variability in tracheostomy tube and tracheostomy suture management (which in turn affects swallowing evaluation) gaining a better understanding of the potential effects of tracheostomy sutures on swallowing function may result in improved patient outcomes.

\section{Conclusion}

No prior studies have formally examined the effect of early tracheostomy suture removal on post-tracheostomy swallowing function. In addition to surgical dissection and the tracheostomy tube itself, the sutures may anchor hyolaryngeal excursion by tethering superficial infrahyoid muscles and underlying soft tissues, thus further reducing hyolaryngeal excursion and resulting in swallowing dysfunction. The presented clinical case raises a question of whether early tracheostomy suture removal can improve post-tracheostomy swallowing function. It highlights the need for further investigation of this issue to prevent post-tracheostomy dysphagia and to create uniform guidelines for appropriate timing of tracheostomy suture removal.

\section{References}

1. Manikantan K, Khode S, Sayed SI, et al. (2009) Dysphagia in head and neck cancer. Cancer Treat Rev 35: 724-732.

2. Bonanno PC (1971) Swallowing dysfunction after tracheostomy. Ann Surg 174: 29-33.

3. Kim Y, McCullough $G$ (2008) Maximum hyoid displacement in normal swallowing. Dysphagia 23: 274-279.

4. Brates D, Molfenter SM, Thibeault SL (2019) Assessing hyolaryn- 
geal excursion: Comparing quantitative methods to palpation at the bedside and visualization during videofluoroscopy. Dysphagia 34: 298-307.

5. Restrepo-Agudelo S, Roldan-Vasco S, Ramirez-Arbelaez L, et al. (2017) Improving surface EMG burst detection in infrahyoid muscles during swallowing using digital filters and discrete wavelet analysis. J Electromyogr Kinesiol 35: 1-8.

6. Nguyen J, Duong H (2020) Anatomy, head and neck, sternohyoid muscle. In: StatPearls. Treasure Island (FL): StatPearls Publishing.

7. Goff D, Patterson J (2019) Eating and drinking with an inflated tracheostomy cuff: A systematic review of the aspiration risk. International Journal of Language \& Communication Disorders.
54: $30-40$.

8. Terk A, Leder S, Burrell M (2007) Hyoid bone and laryngeal movement dependent upon presence of a tracheotomy tube. Dysphagia 22: 89-93.

9. Schaetzel S, Juern J, Kiehl K, et al. (2013) The effect of suturing on force for dislodgement of tracheostomy tubes: Medial versus lateral sutures. J Trauma Acute Care Surg 75: 492-495.

10. Dixon LM, Mascioli S, Mixell JH, et al. (2018) Reducing tracheostomy-related pressure injuries. AACN Adv Crit Care 29: 426-431.

11. O'Toole TR, Jacobs N, Hondorp B, et al. (2017) Prevention of tracheostomy-related hospital-acquired pressure ulcers. Otolaryngol Head Neck Surg 156: 642-651. 